\title{
EXERCíCIOS BASEAdOS NO MÉTOdO PILATES NO TRATAMENTO DE PORTADORAS DE FIBROMIALGIA: RELATO DE CASOS
}

Raissa Puzzi Ladvig, Maria Rita Masselli, Dalva Minonroze Albuquerque Ferreira

Universidade Estadual Paulista - UNESP, Faculdade de Ciências e Tecnologia - FCT, Presidente Prudente, SP. e-mail: ladvigra@hotmail.com

\section{RESUMO}

O estudo analisou os efeitos do tratamento pelo Método Pilates (MP) em mulheres com fibromialgia. Quatro pacientes com diagnóstico de fibromialgia foram avaliadas antes e depois de receberem 20 sessões de exercícios baseados no MP, sendo utilizados na avaliação: Questionário de Impacto da Fibromialgia, palpação dos tender-points, Questionário de Qualidade de Vida (SF-36) e Teste Terceiro Dedo-Chão. Os exercícios fram realizados inicialmente no solo, e com bola na medida em que os pacientes estivessem preparados para maior grau de dificuldade. Os resultados apresentaram, no QIF, sucesso das pacientes em todos os domínios, com exceção do domínio ansiedade apenas para uma paciente. A melhora geral também vale para os resultados no número de tender-points, teste do terceiro dedochão e o SF-36. Os exercícios baseados no Método Pilates foram eficazes no tratamento das quatro pacientes com a Síndrome da Fibromialgia, principalmente nos aspectos da qualidade de vida, dor e flexibilidade.

Palavras-chave: fibromialgia, modalidades de fisioterapia, qualidade de vida.

\section{EXERCISES BASED ON THE PILATES METHOD IN TREATING FIBROMYALGIA PATIENTS: CASE REPORTS}

\section{ABSTRACT}

The study examined the effects of treatment with Pilates (MP) in women with fibromyalgia. Four patients diagnosed with fibromyalgia were evaluated before and after receiving 20 sessions of exercises based on the MP, being used in the evaluation: Fibromyalgia Impact Questionnaire, palpation of tender points, Quality of Life Questionnaire (SF-36) and Test Third Finger-floor. The drills were initially performed on the ground, and in that ball patients were prepared for greater degree of difficulty. The results presented in QIF, success of patients in all areas, except the area just anxiety for a patient. The overall improvement also applies to the results in the number of tender points, the third finger-ground test and the SF-36. The exercises based on the Pilates Method were effective in the treatment of four patients with Fibromyalgia Syndrome, particularly in aspects of quality of life, pain and flexibility.

Keywords: fibromyalgia, physical therapy modalities, quality of life.

\section{INTRODUÇÃO}

A Síndrome da Fibromialgia (SFM), determinada por um estudo brasileiro, tem sua prevalência de $2,5 \%$ na população, sendo a maioria do sexo feminino, das quais $40,8 \%$ entre 35 e 44 anos de idade. A patologia é uma condição comumente observada na prática clínica diária e uma das principais causas de consultas referentes ao sistema musculoesquelético, sendo ainda considerada o segundo distúrbio reumatológico mais encontrado, superada apenas pela osteoartrite'.

Embora reconhecida há muito tempo, a Fibromialgia tem sido seriamente pesquisada somente há três décadas. Pouco ainda é conhecido sobre sua etiologia e patogênese, e até o momento, não existem tratamentos que sejam considerados muito eficazes ${ }^{2}$. Ela é uma síndrome complexa, não inflamatória, caracterizada por dor musculoesquelética difusa e crônica, bem como sítios dolorosos específicos à palpação, denominados tender-points (pontos dolorosos), mas sem anormalidades estruturais na musculatura ${ }^{3}$. Outros aspectos sintomáticos são fadiga, rigidez matinal, distúrbios do padrão de sono, prejuízos cognitivos, depressão, ansiedade, sensação de incapacidade, câimbras e algumas vezes, queixas vagas de sensação de edema em partes moles ou parestesias que podem variar com a hora do dia, tipo de atividade e clima. A SFM acomete em maior parte a população feminina, geralmente entre 30 e 50 anos de idade, podendo manifestar-se em crianças, adolescentes e indivíduos idosos ${ }^{4}$.

A SFM apresenta impacto negativo na vida das pacientes, com redução da capacidade funcional e piora do estado geral de saúde. Pacientes fibromiálgicas apresentam níveis mais 
altos de dor, o que acarreta limitações funcionais e físicas, menor flexibilidade, falta de condicionamento aeróbico e menor capacidade para realizar atividades de vida diária ${ }^{5}$.

Para o diagnóstico da SFM há alguns critérios baseados nas características clínicas, são eles, dor generalizada persistente no mínimo por três meses, bilateral, acima e abaixo da cintura e presença de dor à palpação digital em no mínimo 11 dos 18 tender-points ${ }^{6}$.

$\mathrm{O}$ tratamento deve incluir medicamentos, visando proporcionar um alívio da dor e dos sintomas. Além de adjuvantes, como atividades físicas regulares, que aumentam os níveis hormonais ligados a nocicepção (encontrados diminuídos no fibromiálgico) e também proporcionam a elevação da temperatura corporal agindo como efeito tranquilizante; psicoterapia, biofeedback, balneoterapia, hidroterapia, eletroterapia, acupuntura também são recomendados ${ }^{7}$. Entre essas opções de tratamento está o Método Pilates (MP), que consiste em uma série de exercícios para melhorar a flexibilidade, consciência corporal, equilíbrio e força, e também reduz a dor por meio de relaxamento e alongamento ${ }^{8}$.

O Método Pilates estimula a circulação, melhora o condicionamento físico, a flexibilidade, o alongamento e o alinhamento postural, além de proporcionar melhorar dos níveis de consciência corporal e da coordenação motora. Tais benefícios ajudam a prevenir lesões e proporcionar um alívio de dores crônicas, porém os mesmos dependem da execução de seus exercícios com fidelidade aos seus princípios. Por se tratar de uma atividade que não impõe desgaste articular e cujo número de repetições de cada exercício é reduzido, promove-se a prevenção e/ou tratamento de certas patologias ${ }^{9}$. De forma mais completa, Robinson e Napper ${ }^{10}$, descrevem o Método Pilates com oito princípios básicos, são eles: concentração (concentração no em cada movimento executado), coordenação (que estimulará um canal de comunicação de duas vias com o objetivo de alimentar o bom funcionamento do cérebro e dos padrões adequados de estímulo muscular), centralização (exercitar-se a partir da força dos músculos profundos denominados de power house), respiração (que objetiva a expansão da caixa torácica), alinhamento (essencial para restabelecer o equilíbrio muscular no corpo), vigor (evita o desperdício de energia com tensões desnecessários), movimentos fluidos (reeducar o corpo a se mover de forma correta novamente) e o relaxamento (aprender a identificar e liberar as áreas de tensão do corpo).

O método apresenta diversas variações, podendo ser realizado por pessoas que buscam um melhor condicionamento físico, até portadores de dores crônicas e doenças reumatológicas ${ }^{9}$. Isto é possível devido ao fato dele poder ser desenvolvido atendendo as necessidades específicas de cada praticante, adequadas com seu biotipo, estado de condicionamento físico e sua capacidade, não havendo contra-indicações. Seus exercícios podem ser adaptados de acordo com a patologia e dificuldades do praticante, sendo assim eles proporcionam diversas variedades que se encaixam muito bem nas necessidades físicas dos mesmos $^{11}$. O MP pode ser praticado por pessoas com SFM, pois se concentra em contrações isométricas e causa menos fadiga que os exercícios aeróbicos ${ }^{12}$.

É preciso pesquisar novos métodos de tratamento para a SFM, como a utilização de exercícios baseados nos princípios do Método Pilates, sugerindo que estes proporcionem progressivo recondiciona-mento neuromusculoesquelético.

O objetivo do estudo foi analisar os efeitos dos exercícios baseados no Método Pilates, aplicados no tratamento de portadoras da Síndrome da Fibromialgia e comparar os resultados previamente e após a aplicação do método.

\section{MATERIAL E MÉTODOS}

Foi realizado um estudo de casos com intervenção em caráter analítico e comparativo, composto por quatro pacientes com encaminhamento médico diagnosticado com SFM, do gênero feminino, entre 30 e 60 anos de idade, para tratamento com exercícios baseados no Método Pilates. O número foi limitado a quatro indivíduos para a intervenção, pois o método exige um monitoramento quase exclusivo durante a execução dos exercícios. Foram realizadas 20 sessões de uma hora cada, sendo a frequência de três vezes na semana, num Centro de Fisioterapia e Reabilitação na UNESP.

Os critérios de inclusão abrangeram diagnóstico clínico da Síndrome da Fibromialgia, presença de um nível de entendimento para os procedimentos que foram realizados, disponibilidade e interesse para a participação, e 
não estar praticando atividades físicas regularmente durante o período do estudo. 0 presente estudo foi aprovado pelo Comitê de Ética e Pesquisa no Processo no 525.032, tendo as participantes, concordado e assinado o Termo de Consentimento Livre e Esclarecido antes da iniciação da pesquisa.

Para análise e comparação dos resultados, previamente e após aplicação dos exercícios baseados no Método Pilates, foram utilizadas as seguintes medidas de avaliação: Questionário de Impacto da Fibromialgia (QIF), validado por Marques et $a l_{.}{ }^{13}$; verificação dos pontos sensíveis a digito-pressão, os tenderpoints, validado por Haun et al. ${ }^{14}$; Questionário de Qualidade de Vida (SF-36), validado por Ciconelli et $a l . .^{15}$ e o Teste Terceiro Dedo-Chão (avalia flexibilidade da cadeia mestra posterior), validado por Perret et $a l^{16}$.

O Questionário de Impacto da Fibromialgia (QIF) consiste em 10 domínios sendo eles e suas respectivas pontuações: capacidade funcional (0-30); sentiu-se bem (0-7); faltas ao trabalho (0-7); capacidade em realizar o serviço (0-10); dor (0-10); fadiga (0-10); cansaço matinal (0-10); rigidez (0-10); ansiedade (0-10); depressão (0-10). Todas as questões apresentam melhores resultados se estiverem mais próximas do valor zero, sendo a única exceção, o segundo domínio, em que, quanto mais próximo do valor zero, pior o resultado.

O tender-points (quanto menos pontos, melhor), ao todo, são 18 tender-points que são pontos preestabelecidos bilateralmente nos locais: região occipital, borda médio-superior do trapézio, músculo supraespinhoso, quadrante superior-externo do glúteo, grande trocanter, região equivalente entre os espaços vertebrais de C5-C7, junção da segunda costela, 2 centímetros abaixo do epicôndilo lateral e borda medial do joelho; notando-se dor à palpação em pelo menos 11 dos 18 tender-points.

O Questionário de Qualidade de Vida (SF36) consiste em 36 domínios englobados em oito componentes: capacidade funcional (10 itens), aspectos físicos (4 itens), dor ( 2 itens), estado geral de saúde (5 itens), vitalidade (4 itens), aspectos sociais ( 2 itens), aspectos emocionais ( 3 itens), saúde mental (5 itens), e mais uma questão de avaliação comparativa entre as condições de saúde atual e de um ano atrás. Cada componente varia de zero a cem, sendo zero o pior e cem o melhor escore.
No Teste do Terceiro Dedo-Chão, o participante, a partir da posição bípede realiza a flexão anterior do tronco, sem fletir os joelhos ou deslocar a pelve posteriormente, levando as mãos em direção ao chão e medir em centímetros a distância do terceiro dedo até o chão. Ele fornece indicações sobre a flexibilidade da cadeia muscular posterior, sendo utilizado como parâmetro de evolução para o tratamento proposto, aproximar ou chegar a zero centímetros do chão.

No estudo, os exercícios baseados no MP escolhidos foram realizados no solo e com bola (nas posições deitado, sentado, quatro apoio e em pé), e foram evoluídos na medida em que as pacientes estavam preparadas para maior grau de dificuldade.

Os exercícios aplicados foram extraídos da apostila elaborada por Paccini ${ }^{17}$, intitulada Pilates: Consciência Corporal e Condicionamento Físico. Iniciou-se o tratamento com exercícios básicos no solo como Side Kick Side Passé (série de chutes), Saw (serra), Mermaid (sereia), Swan Dive Prep. (preparação para o mergulho do cisne), Double Leg Kick (chute duplo), Shoulder Bridge (ponte ombros), Roll Down (rolando para baixo) e Double Leg Strech (duplo alongamento de pernas). E básicos com a bola como: Articulação do Quadril, Abdominal com Alongamento, Cisne, Rolar para Frente, Semi Rolamento, Rotação de Tronco, Ponte e Extensão de tronco. Todos os exercícios eram executados em duas séries de dez repetições, com pequenos intervalos entre a troca de exercícios.

Visando a evolução dos exercícios para as pacientes foram acrescentados exercícios de nível intermediário no solo como: Swan Dive (mergulho do cisne), Pyramid (pirâmide), Roll Over (rolando para trás) e Shoulder Bridge 2 (ponte de ombros- variação). E de nível intermediário com a bola como: Concha, Inclinação Lateral, Flexão de Braço e Extensão de tronco 2. Ambas as séries de exercícios foram modificados e adaptados quando necessário.

\section{RESULTADOS}

Participaram deste estudo quatro mulheres com diagnóstico clínico de Fibromialgia, usuárias do serviço de saúde da UNESP, todas elas com frequência regular nas sessões, dispostas e motivadas para o tratamento.

Na tabela 1 estão os resultados obtidos do Questionário de Impacto da Fibromialgia (QIF) 
(todas as questões apresentam melhores resultados se estiverem mais próximas do valor zero, sendo a única exceção, o segundo domínio, em que, quanto mais próximo do valor zero, pior o resultado).

Na tabela 2 estão os dados obtidos do tender-points (quanto menos pontos, melhor), e do Teste Terceiro Dedo-chão (quanto menos centímetros, melhor).

$\mathrm{Na}$ tabela 3 estão os resultados do Questionário de Qualidade de Vida (SF-36) com cada componente variando de zero a cem, sendo zero o pior e cem o melhor escore.

Tabela 1. Valores do QIF nas 4 pacientes de fibromialgia, antes e após a intervenção com o Método Pilates.

\begin{tabular}{lcccccccc}
\hline Questionário do Impacto da & \multicolumn{2}{c}{ Paciente $\mathbf{1}$} & \multicolumn{2}{c}{ Paciente 2 } & \multicolumn{2}{c}{ Paciente 3 } & \multicolumn{2}{c}{ Paciente 4 } \\
\cline { 2 - 9 } Fibromialgia (QIF) & antes & depois & antes & depois & antes & depois & antes & depois \\
\hline Capacidade Funcional & 5 & 4 & 14 & 0 & 11 & 2 & 13 & 4 \\
Sentiu-se bem & 3 & 6 & 0 & 7 & 0 & 4 & 2 & 6 \\
Faltas ao trabalho & 1 & 1 & 1 & 0 & 1 & 0 & 0 & 0 \\
Capacidade em realizar o serviço & 3 & 3 & 5 & 4 & 5 & 0 & 7 & 5 \\
Dor & 7 & 2 & 9 & 4 & 10 & 5 & 8 & 3 \\
Fadiga & 8 & 3 & 7 & 3 & 9 & 4 & 6 & 6 \\
Cansaço Matinal & 5 & 3 & 10 & 3 & 8 & 5 & 8 & 2 \\
Rigidez & 5 & 4 & 7 & 3 & 10 & 3 & 10 & 7 \\
Ansiedade & 10 & 2 & 10 & 3 & 7 & 9 & 10 & 6 \\
Depressão & 5 & 2 & 8 & 4 & 7 & 6 & 8 & 3 \\
\hline
\end{tabular}

Tabela 2. Valores do tender-points (número de pontos de dor) e Teste terceiro dedo-chão $(\mathrm{cm})$ nas 4 pacientes de fibromialgia, antes e após a intervenção com o Método Pilates.

\begin{tabular}{lcccccccc}
\hline & \multicolumn{2}{c}{ Paciente 1 } & \multicolumn{2}{c}{ Paciente 2 } & \multicolumn{2}{c}{ Paciente 3 } & \multicolumn{2}{c}{ Paciente 4 } \\
\cline { 2 - 9 } & antes & depois & antes & depois & antes & depois & antes & depois \\
\hline Tender-Points & 8 & 5 & 15 & 8 & 13 & 8 & 10 & 8 \\
Teste 3 Dedo-Chão(cm) & 22 & 2 & 8 & 0 & 2 & 0 & 11,5 & 0 \\
\hline
\end{tabular}

Tabela 3. Valores do SF-36 nas 4 pacientes de fibromialgia, antes e após a intervenção com o Método Pilates.

\begin{tabular}{|c|c|c|c|c|c|c|c|c|}
\hline \multirow{2}{*}{$\begin{array}{l}\text { Questionário de Qualidade } \\
\text { de Vida (SF-36) }\end{array}$} & \multicolumn{2}{|c|}{ Paciente 1} & \multicolumn{2}{|c|}{ Paciente 2} & \multicolumn{2}{|c|}{ Paciente 3} & \multicolumn{2}{|c|}{ Paciente 4} \\
\hline & antes & depois & antes & depois & antes & depois & antes & depois \\
\hline Capacidade funcional & 80 & 95 & 25 & 55 & 60 & 75 & 45 & 50 \\
\hline Limitação por aspectos físicos & 0 & 75 & 0 & 100 & 25 & 100 & 50 & 50 \\
\hline Dor & 51 & 85 & 10 & 74 & 20 & 61 & 31 & 41 \\
\hline Estado geral de saúde & 57 & 82 & 15 & 40 & 2 & 47 & 52 & 77 \\
\hline Vitalidade & 45 & 70 & 35 & 60 & 15 & 35 & 40 & 50 \\
\hline Aspectos sociais & 62,5 & 100 & 25 & 87,5 & 37,5 & 75 & 12,5 & 62,5 \\
\hline Aspectos emocionais & 0 & 100 & 0 & 100 & 33,3 & 100 & 0 & 33,3 \\
\hline Saúde mental & 68 & 84 & 20 & 56 & 44 & 56 & 44 & 64 \\
\hline
\end{tabular}


$\mathrm{Na}$ literatura há diversos estudos envolvendo a $\mathrm{SFM}^{2,3,4,5}$ e outros abordando o Método Pilates ${ }^{9,11}$, porém, a junção dos dois temas ainda é escasso ${ }^{18,12 .}$

No estudo de $A m o r i m^{18}$, o objetivo era avaliar, em pacientes fibromiálgicas, os efeitos do Método Pilates nas atividades de vida diária (QIF), na dor (escava visual analógica), no número dos tender-points (pela dígito-pressão) e na amplitude de movimento dos ombros e quadris (pela biofotogrametria). $O$ estudo concluiu que o MP foi eficaz na diminuição do impacto da fibromialgia na vida diária, na intensidade da dor e no número dos tender-points, porém não foi eficiente para o ganho da amplitude de movimento da flexão dos ombros e dos quadris. Em contrapartida, o presente estudo, além de ótimos resultados no QIF e tender-points, também obteve resultados satisfatórios para o teste de flexibilidade pelo Teste Terceiro DedoChão.

Altan et $a l .{ }^{12}$, que investigaram a eficácia do método Pilates na dor, funcionalidade e qualidade de vida em 49 pacientes com fibromialgia, consistiu na realização de exercícios do método em sessões de 1 hora, 3 vezes por semana, durante 12 semanas. De acordo com o programa, o estudo sugeriu o Pilates como sendo eficaz e seguro para pacientes com diagnóstico de fibromialgia. Os resultados se equivalem aos encontrados no presente estudo, que também obteve resultados satisfatórios utilizando o MP.

Sendo o Método Pilates considerado uma atividade física que envolve principalmente o alongamento, além do fortalecimento da musculatura, pesquisou-se também estudos que envolvessem atividades voltadas para 0 alongamento no tratamento de pacientes com Fibromialgia. Como no estudo de Matsutani, Assumpção e Marques ${ }^{19}$, que comparou os efeitos dos exercícios aeróbios e do alongamento na dor, número de tender-points, sono, ansiedade e depressão de pacientes com SFM, foi observado que o ganho foi maior no grupo alongamento em relação à dor, número de tender points, sono e depressão ao se comparar com o grupo aeróbio. Já o ganho para ansiedade foi mais importante no grupo aeróbio em comparação ao grupo alongamento. Pode-se concluir que os resultados dos autores se equivalem aos do presente estudo, pois os bons resultados obtidos em relação ao número de tender-points, sono e depressão, foram proporcionados também devido ao alongamento, que é característico no MP.

Em relação a qualidade de vida das pacientes fibromiálgicas, avaliadas no presente estudo através do SF-36, se obteve, no geral, bons resultados em consequência ao tratamento utilizando os exercícios baseados no MP. Um estudo feito para verificar os efeitos da prática constante do Método Pilates na qualidade de vida de seus pacientes, utilizando o SF-36 como método de avaliação, constatou que estes podem melhorar a qualidade de vida de seus praticantes. Seus resultados demonstraram que a capacidade funcional, limitação por aspectos físicos, dor, estado geral de saúde, vitalidade, aspectos sociais e saúde mental, apresentaram diferenças significantes, somente os aspectos emocionais não evidenciou uma diferença significante após a aplicação dos exercícios ${ }^{20}$. Os resultados dos autores em conjunto aos do presente estudo, reforçam a ideia de o método ser eficaz tanto para melhora da qualidade de vida de pacientes com a SFM, quanto para qualquer praticante.

Uma limitação do estudo foi o número pequeno de participantes devido ao Método Pilates exigir atenção especial e individualizada para cada praticante. Apesar da limitação do estudo tentou-se contribuir para o tema, que ainda se apresenta escasso na literatura. Mais estudos sobre o assunto são necessários, com número amostral adequado, para melhor demonstração da eficácia dos exercícios.

\section{CONCLUSÃO}

Os exercícios baseados no Método Pilates foram eficazes no tratamento das quatro pacientes com a Síndrome da Fibromialgia. A qualidade de vida, a dor, e a flexibilidade foram os aspectos que obtiveram maior êxito diante dos exercícios propostos, reflexo disso observa-se em seus resultados, que de maneira geral, fez-se atingir as expectativas e objetivos positivamente.

\section{CONFLITO DE INTERESSE}

Os autores declaram não haver qualquer potencial de conflito de interesse que possa interferir na imparcialidade deste trabalho científico. 


\section{REFERÊNCIAS}

1. Helfenstein Jr M, Goldenfum NA, Siena CAF. Fibromialgia: aspectos clínicos e ocupacionais. Rev Assoc Med Bras. 2012;3(58):358-65. DOI: http://dx.doi.org/10.1590/S0104-

42302012000300018

2. Heymann RE, Paiva ES, Helfentein Jr M, Pollak DF, Martinez JE, Provenza JR et al. Consenso brasileiro do tratamento da fibromialgia. Rev Bras Reumatol. 2010;50(1). DOI: http://dx.doi.org/10.1590/S0482$\underline{50042010000100006}$

3. Leal AS. Análise da atuação da fisioterapia na qualidade de vida de pacientes com fibromialgia: estudo de caso. [monografia] Campina Grande: Universidade Estadual da Paraíba, 2011.

4. Ramiro FS, Junior IL, Silva RCB, Montesano FT, Oliveira NRC, Diniz REAS et al. Investigação do estresse, ansiedade e depressão em mulheres com fibromialgia: um estudo comparativo. Rev Bras Reumatol. 2013;1(54):27-32. DOI: http://dx.doi.org/10.1016/j.rbr.2013.04.006

5. Oliveira RM; Leite ACS; Silva LMS; Almeida PC; Oliveira SKP; Chaves ACP. Comparative analysis of functional capacity among women with fibromyalgia and low back pain. Rev Dor. 2013;1(14). DOI: http://dx.doi.org/10.1590/S1806-

\section{0}

6. Hidalgo DC. Actualización en fibromialgia. Medicina Legal de Costa Rica. 2013;30(1): 83-88.

7. Konrad LM; Lopes AS. Efeito agudo do exercício físico sobre a qualidade de vida de mulheres com a síndrome da fibromialgia. Rev Bras Ativ Fis Saúde. 2005;10(2):59-59

8. Marques D. Análise da capacidade funcional de mulheres idosas praticante do Método Pilates. [monografia] Criciúma: Universidade do Extremo Sul Catarinense, 2012.

9. Comunello JF. Benefícios do Método Pilates e sua aplicação na reabilitação. São Paulo: Instituto Salus; 2011.

10. Robinson L; Napper H. Exercícios inteligentes com Pilates e Yoga. 10.ed. São Paulo: Editora Pensamento; 2011.

11. Miranda LB; Morais PDC. Efeitos do Método Pilates sobre a composição corporal e flexibilidade. Rev Bras Presc Fisiol Exerc. 2009;3(13):16-21.

12. Altan L; Korkmaz N; Bingol U; Gunay B. Effect of Pilates training on people with fibromyalgia syndrome: a pilot study. Arch Phys Med Rehabil. 2009;90(12):1983-8.

DOI:

http://dx.doi.org/10.1016/j.apmr.2009.06.021

13. Marques AP; Santos AMB; Assumpção A; Matsunami LA; Lage LV; Pereira CAB. Validação da versão brasileira do Fibromyalgia Impact Questionnaire (FIQ). Rev Bras Reumatol. 2006;46(1):24-316. DOI:
14. Haun MVA.; Ferraz MB; Pollak DF. Validação dos critérios do Colégio Americano de Reumatologia (1990) para classificação da Fibromialgia, em uma população brasileira. Rev Bras Reumatol. 1999;39(4):21-30.

15. Ciconelli RM; Ferraz MB; Santos W; Meinão I; Quaresma MR. Tradução para a língua português e validação do questionário genérico de avaliação de vida SF-36 (Brasil SF-36). Rev Bras Reumatol. 1999;39(3): 143-150.

16. Perret C; Poiraudeau S; Fermanian J; Colau MML; Benhamou MAM; Revel M. Validity, reliability, and responsiveness of the fingertip-to-floor test. Arch Phys Med Rehabil. 2001;82(15):66-70. DOI: http://dx.doi.org/10.1053/apmr.2001.26064

17. Paccini C. Pilates Consciência Corporal e Condicionamento Físico. [apostila] Presidente Prudente, 2012.

18. Amorim NMF. Efeito do método Pilates como tratamento adjuvante em pacientes portadores de fibromialgia. [dissertação]. Maranhão: Universidade Federal do Maranhão, 2009.

19. Matsutani LA; Assumpção A; Marques AP. Exercícios de alongamento muscular e aeróbico no tratamento da fibromialgia: estudo piloto. Fisioter Mov. 2012;25(2):411-8. DOI: http://dx.doi.org/10.1590/S0103-

51502012000200019

20. Carvalho CF; França AA; Beneli LM. Análise da qualidade de vida em pessoas que praticam os exercícios de método Pilates. Anuário de Produção de Iniciação Científica Discente. 2011;14(23):97-104.

Recebido para publicação em 04/01/2015

Revisado em 15/08/2015

Aceito em 22/01/2016 